\title{
Wiener and Hyper-Wiener Indices of Unitary Addition Cayley Graphs
}

\author{
C. Thilaga, P.B. Sarasija
}

\begin{abstract}
A topological index is a number associated to a graph. In chemical graph theory the Wiener index of a graph $G$, denoted by $W(G)$ is the sum of the distance between all (unordered) pairs of vertices of $G$. That is, $W(G)=\sum_{i<j} d\left(u_{i}, u_{j}\right)$ , where $d\left(u_{i}, u_{j}\right)$ is the shortest distance between the vertices. $u_{i}$ and $u_{j}$.The Hyper-Wiener Index $W W(G)$ is the generalization of the Wiener index. The Hyper-Wiener Index of a graph $G$ is, WW $(G)=\sum_{i<j}\left(d\left(u_{i}, u_{j}\right)+d\left(u_{i}, u_{j}\right)^{2}\right)$. The unitary addition Cayley graph $G_{n}$ has a vertex set $Z_{n}=\{0,1, \ldots, n-1\}$ and the vertices $u$ and $v$ are adjacent if $g c d(u+v, n)=1$. In this paper Wiener index and Hyper Wiener indices of Unitary addition Cayley graph $G_{n}$ is computed.
\end{abstract}

Keywords--- Unitary Cayley Graphs, Unitary addition Cayleygraphs, Wiener Index and Hyper, Wiener Index.

AMS Classification 2010--- 05C50, $05 C 78$.

\section{INTRODUCTION}

In this paper, we consider simple connected graph $\mathrm{G}=$ $(\mathrm{V}, \mathrm{E})$ with $\mathrm{n}$ vertices and $\mathrm{m}$ edges. The topological index is a numeric quantity associated with a graph, and is invariant under a graph automorphism. Most of the proposed topological indices are related either through a vertex adjacency relationship or through the topological distances in $\mathrm{G}$.

The first topological index is a Wiener Index [ 1,4 ]. This index is used in Chemistry.

We denote the shortest distance between the vertices $u_{i}$ and $\mathrm{u}_{\mathrm{j}}$ with $\mathrm{d}\left(\mathrm{u}_{\mathrm{i}}, \mathrm{u}_{\mathrm{j}}\right)$.

The Wiener index $W(G)$ is equal to the sum of the distances between all pairs of vertices of $G$.

That is

$$
\mathrm{W}(\mathrm{G})=\sum_{i<j} d\left(\boldsymbol{u}_{i}, u_{j}\right)=\frac{1}{2} \sum_{i=1}^{n} \sum_{j=1}^{n} d\left(u_{i}, u_{j}\right),
$$

The Hyper-Wiener Index WW [6 ] is the generalization of the Wiener index. The Hyper-Wiener Index of the connected graph G is, WW $(\mathrm{G})=\sum_{i<j}\left(d\left(u_{i}, u_{j}\right)+d\left(u_{i}, u_{j}\right)^{2}\right)$,

Let $\mathrm{G}$ be a group, and let $S \subseteq G$ be a set of group elements such that the identity element $I \notin S$.

The Cayley graph associated with $(\mathrm{G}, \mathrm{S})$ is then defined as the directed graph having one vertex associated with each group element and directed edges $(\mathrm{g}, \mathrm{h})$, whenever $\mathrm{gh}^{-1} \in S$. [2]

Let $\mathrm{G}$ be an abelian group and $\mathrm{S}$ be a subset of the addition Cayley graph $\mathrm{G}^{\prime}=\operatorname{Cay}^{+}(G, \mathrm{~S})$ is the graph having the vertex set $\mathrm{V}\left(\mathrm{G}^{\prime}\right)=G$ and the edge set $\mathrm{E}\left(\mathrm{G}^{\prime}\right)=\{\mathrm{uv}: \mathrm{u}+$ $\mathrm{v} \in \mathrm{S}\}$, where $\mathrm{u}, \mathrm{v} \in \mathrm{G}[3,5]$

For a positive integer $n>1$, the unitary addition Cayley graph $G_{n}$ is the graph whose vertex set is $Z_{n}$, the integers modulo $n$ and if $U_{n}$ denotes the set of all units of the ring $Z_{n}$, then two vertices $u$ and $v$ are adjacent if and only if $u+v \in$ $\mathrm{U}_{\mathrm{n}}$. The unitary addition Cayley graph $\mathrm{G}_{\mathrm{n}}$ is also defined as, $\mathrm{G}_{\mathrm{n}}=\mathrm{Cay}^{+}\left(\mathrm{Z}_{\mathrm{n}}, \mathrm{U}_{\mathrm{n}}\right)$. Let $\phi(\mathrm{n})$ denotes the Euler function. For several properties of addition Cayley graphs refer, [2] . Also refer [8].

In this paper, we obtain the Wiener and hyper-Wiener indices of Unitary addition Cayley graphs $G_{n}$. Let us see the following lemma which has its inevitable usage in the proof of theorem that follows.

Lemma 1. [7 ]

Denote $F_{n}(s)(n \geq 2)$, the number of solutions of the congruence $x+y=s \bmod n, x \in U_{n}, y \in U_{n}$ and for integers $\mathrm{n}$ and prime $\mathrm{p}$. Then $\mathrm{F}_{\mathrm{n}}(\mathrm{s})=\mathrm{n} \prod_{p / n}\left(1-\frac{g(p)}{p}\right)$,

$$
\text { where } \mathrm{g}(\mathrm{p})= \begin{cases}1, & \text { if } p \text { divides } s \\ 2, & \text { if } p \text { doesnot divides } s\end{cases}
$$

Lemma 2.[ 3 ]

The unitary addition Cayley graph $\mathrm{G}_{\mathrm{n}}, \mathrm{n} \geq 2$ is bipartite iff $\mathrm{n}$ is either even or $\mathrm{n}=3$.

\section{WIENER INDEX OF UNITARY ADDITION CAYLEY GRAPHS}

\section{Theorem 2.1}

The number of common neighbors of distinct vertices $\mathrm{u}, \mathrm{v}$ in the unitary addition Cayley graph $G_{n}$ is given by $F_{n}(a+b)$.

\section{Proof}

Let $\mathrm{u}, \mathrm{v}, \mathrm{w}$ be the elements of in the vertex set $V(G)$.Vertex ' $w$ ' is a common neighbor of $u$ and $v$ if and only if $\operatorname{gcd}(\mathrm{u}+\mathrm{w}, \mathrm{n})=\operatorname{gcd}(\mathrm{v}+\mathrm{w}, \mathrm{n})=1$, then there exist unique $\mathrm{x}, \mathrm{y} \in \mathrm{Z}_{\mathrm{n}}$ such that $\mathrm{u}+\mathrm{w} \equiv \mathrm{x} \bmod \mathrm{n}, \mathrm{v}+\mathrm{w}=\mathrm{y} \bmod$ n.Now $\mathrm{w} \equiv x-u \equiv y-v$ becomes a common neighbor if and only if $\mathrm{x}-\mathrm{y}=\mathrm{u}-\mathrm{v} \bmod \mathrm{n}$.

\section{Theorem 2.2.}

If $\mathrm{G}_{\mathrm{n}}$ is the Unitary addition Cayley graph, then the Wiener index of $G_{n}$ is 


$\mathrm{W}\left(\mathrm{G}_{\mathrm{n}}\right)=$
$\left\{\begin{array}{lr}\frac{n^{2}-1}{2}, & \text { if } n \text { is a primenumber } \\ \frac{4}{3} n^{2}-n, & \text { if } n=2^{\alpha} \alpha>1 \\ \frac{5}{4} n^{2}-n \phi(n)-n, & \text { if } n \text { is evenand odd prime divisor } \\ (n-1)\left(n-\frac{\phi(n)}{2}\right), & \text { if } n \text { is odd but not prime }\end{array}\right.$

\section{Proof}

Suppose $\mathrm{n}$ is a prime number, then $\mathrm{U}_{\mathrm{n}}=\{1,2, \ldots,(\mathrm{n}-1)\} .0$ is adjacent to all vertices and

$\mathrm{u} \in \mathrm{U}_{\mathrm{n}}$ is adjacent to (n-2) vertices. Hence the distance between any two vertices $u$ and

$\mathrm{v}$ are either one or two. Here $\frac{(n-1)}{2}$ vertices have distance 2 and $\frac{(n-1)^{2}}{2}$ vertices have

distance 1, Therefore $\mathrm{W}\left(\mathrm{G}_{\mathrm{n}}\right)=\frac{(\varphi(n))^{2}}{2} \times 1+\frac{\varphi(n)}{2} \times 2=$ $\frac{n^{2}-1}{2}$

When $\mathrm{n}=2^{\alpha}, \alpha>1, \mathrm{G}_{\mathrm{n}}$ is complete bipartite with vertex partition $\mathrm{V}\left(\mathrm{G}_{\mathrm{n}}\right)$ as the union

of $V_{1}\left(G_{n}\right)=\{0,2, \ldots,(n-2)\}$ and $V_{2}\left(G_{n}\right)=\{1,3, \ldots$, (n-

1) $\}$, then it is clear that $d(u, v)=1$ or

$\mathrm{d}(\mathrm{u}, \mathrm{v})=2$. Therefore $\mathrm{W}\left(\mathrm{G}_{\mathrm{n}}\right)=\frac{4}{3} n^{2}-n$.

Now, consider $\mathrm{n}$ as even and has an odd prime divisor $\mathrm{p}$, where $n \neq 2^{\alpha} \alpha>1$.

$G_{n}$ is bipartite with vertex set $V\left(G_{n}\right)$ as the union of $V_{1}\left(G_{n}\right)$ and $V_{2}\left(G_{n}\right)$.For calculating Wiener index of $G_{n}$, first, we find $\mathrm{d}(\mathrm{u}, \mathrm{v})$ as either $\mathrm{u} \in V_{1}\left(G_{n}\right)$ or $\mathrm{u} \in V_{2}\left(G_{n}\right)$

Case (i): Consider $\mathrm{u} \in V_{1}\left(G_{n}\right)$ and $\mathrm{v} \in V_{1}\left(G_{n}\right)$.

Clearly $\mathrm{u}$ and $\mathrm{v}$ are not adjacent. Hence by Theorem 2.1 there exist a common neighbor.

Therefore $\mathrm{d}(\mathrm{u}, \mathrm{v})=2$

Case (ii): Consider $\mathrm{u} \in V_{1}\left(G_{n}\right)$ and $\mathrm{v} \in V_{2}\left(G_{n}\right)$. Then there exist $\emptyset(n)$ neighbors of $\mathrm{u} \in V_{2}\left(G_{n}\right)$.

Now take $\mathrm{V}_{2}\left(\mathrm{G}_{\mathrm{n}}\right)=\mathrm{S} \cup \mathrm{T}$, where $\mathrm{S}=\left\{\mathrm{v} \in \mathrm{V}_{2}\left(\mathrm{G}_{\mathrm{n}}\right)\right.$; uve $\left.\mathrm{E}\left(\mathrm{G}_{\mathrm{n}}\right)\right\}$ and

$\mathrm{T}=\left\{\mathrm{v} \in \mathrm{V}_{2}\left(\mathrm{G}_{\mathrm{n}}\right) ; \mathrm{uv} \notin \mathrm{E}\left(\mathrm{G}_{\mathrm{n}}\right)\right\}$.Clearly, for $\mathrm{u} \in V_{1}\left(G_{n}\right)$ and $\mathrm{v} \in \mathrm{S}, \mathrm{d}(\mathrm{u}, \mathrm{v})=1$.

Let $\mathrm{v} \in T \mathrm{u}$ and $\mathrm{v}$ are not adjacent. Therefore $\mathrm{w} \in S \subset$ $V_{2}\left(G_{n}\right)$. Then $u w \in E\left(G_{n}\right)$. Now $v$ and $\mathrm{w}$

are odd then there exist a common neighbor $\mathrm{x}$ to $\mathrm{v}$ and $\mathrm{w}$. Hence $\mathrm{d}(\mathrm{u}, \mathrm{v})=3$.

Similarly the case of $\mathrm{u} \in V_{2}\left(G_{n}\right) \cdot \mathrm{W}\left(G_{n}\right)=\frac{5}{4} n^{2}-n \emptyset(n)-$ $n$.

Suppose $\mathrm{n}$ is odd, Suppose $\mathrm{n}$ is odd and a non-prime number .Let $\mathrm{p}_{1}, \mathrm{p}_{2} \ldots \mathrm{p}_{\mathrm{t}}$ be the different prime divisors of $\mathrm{n}$, $n=P_{1}^{r_{1}} \times P_{2}^{r_{2}} \times \ldots \ldots \times P_{t}^{r_{t}}$ and $\mathrm{p}_{\mathrm{i}} \neq 2,1 \leq i \leq t$. All the vertices are either adjacent or there exists a common neighbor to every pair of distinct vertices. Hence $\mathrm{d}(\mathrm{u}, \mathrm{v})=1$ or $2 \mathrm{~W}\left(\mathrm{G}_{\mathrm{n}}\right)=\frac{(n-1) \varphi(n)}{2} \times 1+\frac{(n-1)(n-\varphi(n)}{2} \times 2=(\mathrm{n}-1)$ $\left(\mathrm{n} \frac{\varphi(n)}{2}\right)$.

\section{HYPER-WIENER INDEX OF UNITARY ADDITION CAYLEY GRAPHS \& RESULTS}

Theorem 3.1

If $G_{n}$ is the Unitary addition Cayley graph, then the Hyper

- Wiener index is given by

$\mathrm{WW}\left(\mathrm{G}_{\mathrm{n}}\right)=$

$\begin{cases}(n-1)(n+2), & \text { if nis a prime number } \\ 2\left(n^{2}-\frac{3 n}{2}\right) & \text { if } n=2^{\alpha} \alpha>1 \\ \frac{1}{2} n(9 n-10 \phi(n)-6), & \text { if nis evenand odd prime divisor } \\ (n-1)(3 n-2 \phi(n)), & \text { if nis odd but not prime }\end{cases}$

Proof

The proof is quite direct from the proof of Theorem 2.2. That is in the Hyper-Wiener index the distance between the vertices $\mathrm{u}$ and $\mathrm{v}$ is $\left[\mathrm{d}(\mathrm{u}, \mathrm{v})+\mathrm{d}(\mathrm{u}, \mathrm{v})^{2}\right]$.

\section{CONCLUSION}

Wiener Index is the first topological index. Hyper Wiener index is the generalization of the Wiener index. In this paper Wiener index and Hyper Wiener indices of Unitary addition Cayley graph $\mathrm{G}_{\mathrm{n}}$ is computed.

\section{ACKNOWLEDGEMENT}

We are thankful to those who provide their guidance through this work.

\section{REFERENCES}

1. Ante Graovac, Tomaž Pisanski, On the Wiener index of a graph, Journal of Mathematical Chemistry Vol.8,1, pp.53-62.

2. N. Biggs, Algebraic graph theory. Second Edition Cambridge Mathematical Library. Cambridge University Press, 1993.

3. Deepa Sinha, PravinGarg and Anjali Singh, .Some properties of unitary addition Cayley graph Centre for Mathematical sciences, Banasthali University.

4. A. Graovac, T. Pisauski, On the Weiner Index of a graph, J. Math.Chem., 8(1991) 53-662.

5. D. Grynkiewicz, V.F. Lev and O. Serra, The connectivity of addition Cayley graphs, Electron. Notes Discrete Math., 29(2007), 135-139.

6. D. J. Klein, I. lukovits and I. Gutman, On the definition of hyper-Wiener index for cycle-containing structures, J. Chem. Inf. Comput. Sci, 35 (1995) 50-52.

7. W. Klotz and T. Sander, Some properties of unitary Cayley graphs, The Electronic J. Comb. 14 (2007) 1-12.

8. RosansaraPhilipose and P.B.sarasija, Gutman Index and Harary Index of Unitary Cayley Graphs, Int. J. of Engineering and Technology, 7(3) (2018) 1243 1244 